\title{
Detecting the integrated Sachs-Wolfe effect with stacked voids (Corrigendum)
}

\author{
Stéphane Ilić ${ }^{1,2,3}$, Mathieu Langer ${ }^{2,3}$, and Marian Douspis ${ }^{2,3}$ \\ ${ }^{1}$ Institut de Recherche en Astrophysique et Planétologie, 14 avenue Édouard Belin, 31400 Toulouse, France \\ e-mail: stephane.ilic@ias.u-psud.fr \\ 2 Institut d'Astrophysique Spatiale, Université Paris-Sud, UMR8617, 91405 Orsay, France \\ 3 CNRS, 91405 Orsay, France
}

A\&A 556, A51 (2013), DOI: 10.1051/0004-6361/201321150

Key words. cosmic background radiation - dark energy - large-scale structure of Universe - errata, addenda

In our original publication (Ilić et al. 2013), we incorrectly assumed that the voids radii of the publicly available catalogue of Granett et al. (2008) were given in Mpc units, while they actually are provided in units of $\mathrm{Mpc} h^{-1}$. As a consequence, Figs. 1 and 12 of our original paper should be replaced by the ones shown in the present corrigendum (Figs. 1 and 2). Another small mistake was corrected in Fig. 1 where we used the comoving radius of some voids from the Sutter et al. (2012) catalogue instead of their proper radius.
Using the correct radii, the discrepancy between the most significant photometry scale and the size of the voids is less pronounced: the scale of the signal is comprised between 0.7 and 0.9 times the typical size of the voids. The significance of the signal itself does not change significantly and the rest of our discussion remains valid, notably concerning the peculiar features of this signal.

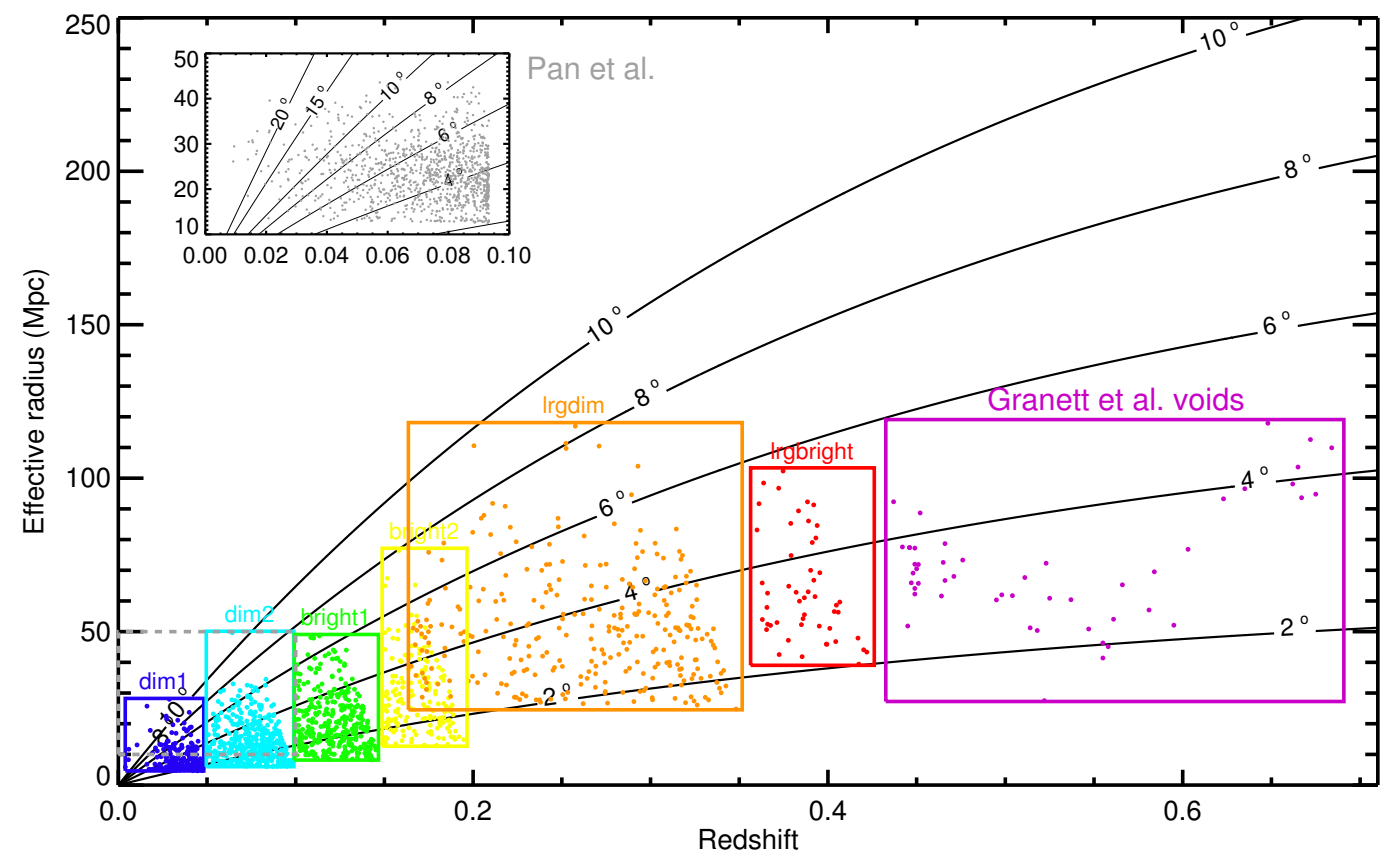

Fig. 1. Effective radii (in Mpc) as a function of redshift for all the voids used in this work. The two catalogues of Granett et al. (2008) and Pan et al. (2012), and the six subsamples of Sutter et al. (2012) are delimited by boxes labelled by their names. The black curves are here to indicate the angular size (in degrees) of a structure in the redshift-radius plane. 
A\&A 572, C2 (2014)
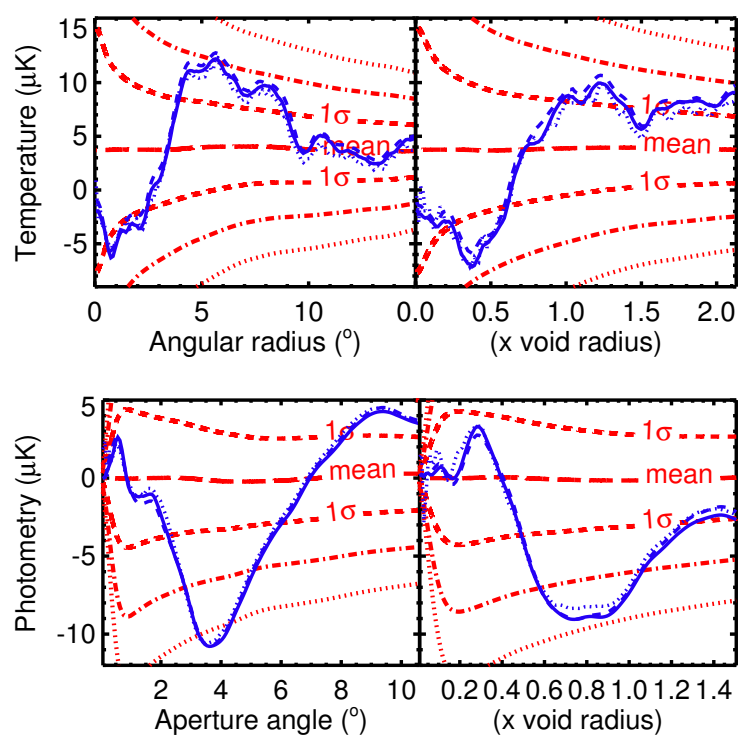

Fig. 2. Temperature (top) and photometry (bottom) profiles for the original (left) and rescaled (right) stacking of Gr08 voids (same conventions as Fig. 7 of our original publication).

\section{References}

Granett, B. R., Neyrinck, M. C., \& Szapudi, I. 2008, ApJ, 683, L99

Ilić, S., Langer, M., \& Douspis, M. 2013, A\&A, 556, A51

Pan, D. C., Vogeley, M. S., Hoyle, F., Choi, Y.-Y., \& Park, C. 2012, MNRAS, 421, 926

Sutter, P. M., Lavaux, G., Wandelt, B. D., \& Weinberg, D. H. 2012, ApJ, 761, 44 